\title{
COMPLEX SYSTEM DESIGN EXPOSURE THROUGH a SATEllite Design Competition
}

\author{
Dario Schor ${ }^{1}$, Kane Anderson ${ }^{1}$, Cody Friesen ${ }^{2}$, Kris Goodmanson ${ }^{3}$, Morgan May $^{3}$, Arash Fazel Darbandi ${ }^{1}$, \\ Witold Kinsner ${ }^{1}$, M.D.(Ron) Britton ${ }^{4}$, and Malcolm Symonds ${ }^{4}$ \\ ${ }^{1}$ Dept. of Electrical \& Computer Engineering, ${ }^{3}$ Dept. of Mechanical Engineering, ${ }^{4}$ Faculty of Engineering \\ ${ }^{2}$ Dept. of Physics \& Astronomy, Faculty of Science \\ University of Manitoba, Winnipeg, MB, Canada R3T 5V6 \\ \{dschor|kinsner\}@ece.umanitoba.ca
}

\begin{abstract}
It is very difficult to teach complex system design within a classroom setting constrained by the number of students, available class time, and type of project feasible within a university course. Therefore, the University of Manitoba is utilizing the Canadian Satellite Design Challenge as an extension of the classroom where students can get exposed to complex systems through the design, implementation, and testing of a triple picosatellite (T-Sat). In this process, the students are exposed not only to many technical challenges, but also to project management that make up the complex project. The team consists of more than 100 undergraduate and graduate students as well as over 50 advisors with various backgrounds.

This paper describes the elements of complex system design experienced throughout the first 18 months of the T-Sat project.
\end{abstract}

Keywords: Complex-system design; complex-system implementation; complex-system management.

\section{INTRODUCTION}

The design of complex engineering systems is characterized by multidisciplinary interdependent teams evaluating trade-offs based on many factors including performance, budgets, timelines, legalities, feasibility, and other factors before selecting the best option for a particular project [1]. This type of holistic experience is rarely taught in a formal setting because of the inherent difficulties in not just presenting all these intertwined concepts and but also the assessment pieces that would capture the student's understanding of the subject accurately. Therefore, in order to expose students to the design of complex systems, many universities and organizations promote engineering design competitions, such as the Canadian Satellite Design Challenge (CSDC) [2]. In the CSDC, teams of undergraduate and graduate students must design, implement, test, and launch an operational triple pico-satellite (T-Sat) carrying at least one scientific experiment. The University of Manitoba (UofM) team competing in this competition consists of more than 100 undergraduate and graduate students from Engineering, Science, Business, Art, and Architecture that are working together in several groups on the T-Sat project [3][4]. They are supported by an infrastructure of over 50 advisors from academia, industry, business, military, and government that provide feedback on the various activities of the project.

This paper presents an overview of the project and some of the lessons learned from the 18 months of the competition highlighting the major aspects of (i) technical complexity, (ii) project management complexity, and (iii) personnel complexity.

\section{T-SAT PROJECT OVERVIEW}

The CSDC was introduced in 2010 to provide students with the opportunity to design, build, and test an operational nanosatellite [2]. The competition ends in September 2012, after which the winning design will be launched into low-Earth-orbit. The UofM team consists of more than 100 students from six faculties and more than 50 advisors with different backgrounds.

The CSDC requirements call for a Sun-synchronous orbit with a negligible eccentricity and altitudes between $600-800 \mathrm{~km}$ above the ground [5][6]. This trajectory causes the satellite to orbit around the Earth every 90-96 minutes with approximately one third of the time spent in eclipse. Simulations show that the satellite will be in contact with the University of Manitoba Satellite Ground Station [7] for close to 30 minutes per day distributed in 4-5 passes with a mean maximum time of 9 minutes.

The satellite bus contains all the components necessary to conduct two on-board experiments. It is $10 \times 10 \times 30 \mathrm{~cm}^{3}$ (as shown on Fig. 1) and has a maximum mass of $4 \mathrm{~kg}$ that meets the requirements for a $3 \mathrm{U}$ PolyPicoSatellite Orbital Deployer (P-POD) used to release the satellite from the launch vehicle [8]. The following sections describe the scientific payloads and subsystems that make up the satellite. 


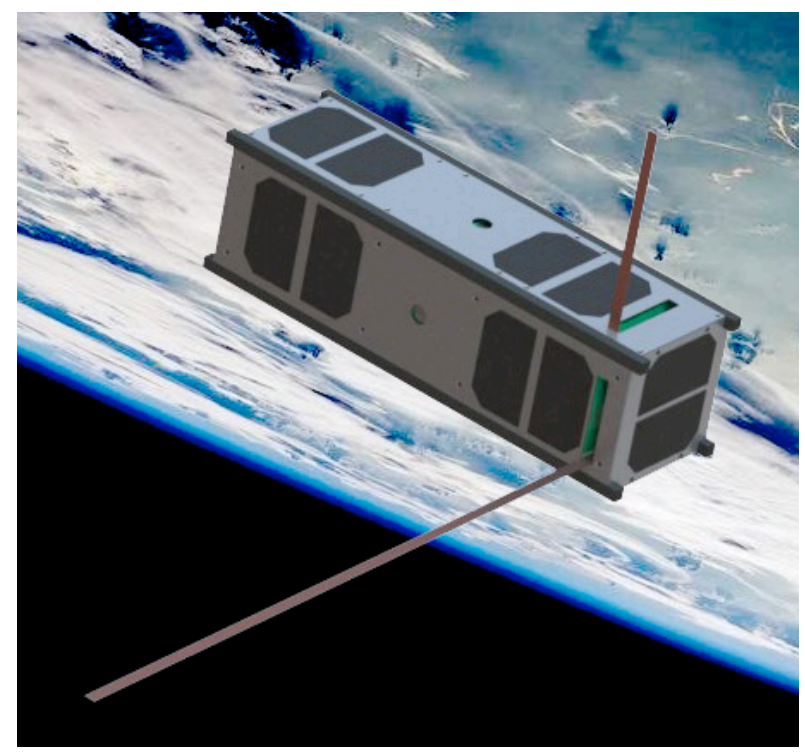

Fig. 1. T-Sat1 in orbit rendition.

\subsection{Scientific Payloads}

The T-Sat project is designed to carry two scientific payloads with on-board experiments for potential use in research and teaching.

Tardigrade Experiment: The primary payload is a biological experiment that tests the survivability of a colony of tardigrades when exposed to the harsh space environment [9]. The tardigrades are launched in a cryptobiotic state and revived in space by inserting water and nutrients into their environment. This process requires collaboration from many groups as solenoid valves are used to control the operation while syringes insert the liquids into the chamber [10].

Spectroscopy Experiment: The second payload is a Solar Spectroscopy experiment that measures the intensity of specific wavelengths emitted by the Sun to compare the results to larger missions [11]. The wavelengths are captured through collimeters mounted on the faces of the spacecraft that transmit the sun radiation to minispectrometers for analysis.

\subsection{Satellite Subsystems}

The satellite bus that carries the scientific payloads is made up of six subsystems, including (i) attitude determination and control (ADC), (ii) communications (COM), (iii) command and data handling $(\mathrm{CDH})$, (iv) power (PWR), (v) Thermal (THM), and (vi) structure (STR), as shown on Fig. 2.

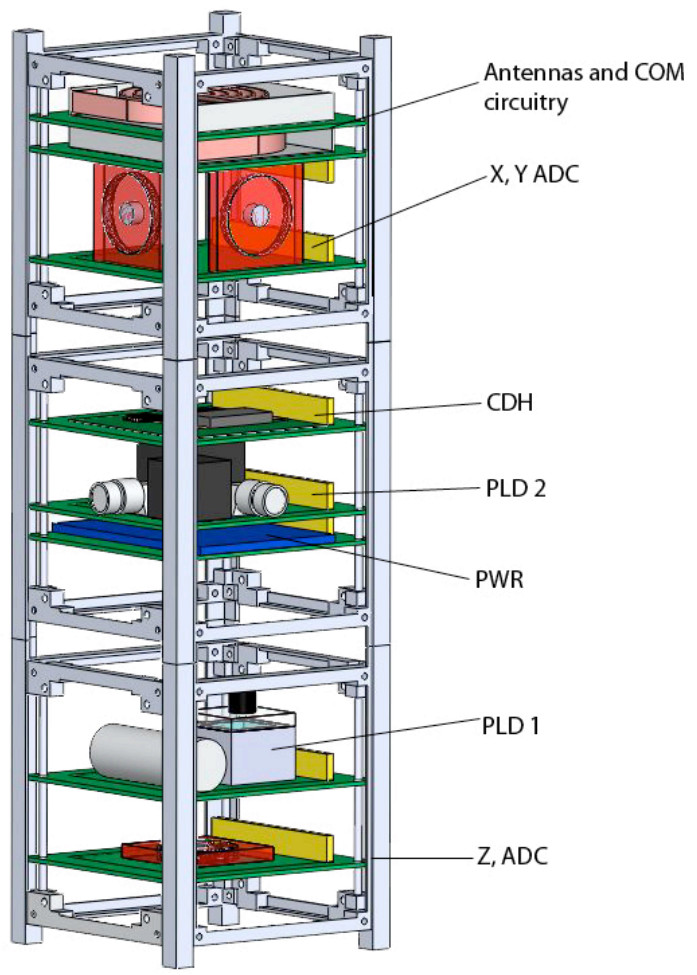

Fig. 5. Layout showing internal components positioning.

Attitude Determination and Control (ADC) Subsystem: This subsystem is responsible for controlling the orientation of the satellite in orbit to slow down the tumbling motion after launch and to point towards the Sun for the spectroscopy payload. The ADC is made up of both hardware and software components to sense the environment, determine the appropriate actions, and exert necessary forces to rotate the satellite. The hardware includes photodiodes to sense the Sun and a magnetometer to measure the Earth's magnetic field [12], while the software is a combination of the B-Dot algorithm and other Sun-pointing algorithms [13].

Communications (COM) Subsystem: The satellite is designed to communicate with the University of Manitoba Satellite Ground Station [7]. Amateur radio bands are used to receive data from the satellite and uplink commands to control the operations [14]. The radio uses a commercial-off-the-shelf transceiver from Yaesu and a custom terminal node controller based on the Texas Instruments MSP430 microprocessor. Special attention is paid to the design of the antennas for the satellite in order to obtain a radiation pattern that allows the satellite to communicate under harsh conditions. 
Command and Data Handling (CDH) Subsystem: All on-board operations are controlled by the microprocessorbased system on the satellite. The hardware for this system consists of a Texas Instruments low-power microprocessor with some additional peripherals to support the operations such as a real-time clock and watchdog timer. The flight software follows a three-layer architecture that abstracts the hardware drivers from the operations of the spacecraft and allows for reusability of the software for future missions. Telemetry and commands are handled through this subsystem [14].

Power (PWR) Subsystem: Solar panels on the exterior faces of the spacecraft collect energy from the Sun to charge a battery that powers the spacecraft operations. In addition, the subsystem features a power distribution and protection system to control the power usage on the spacecraft [15].

Thermal (THM) Subsystem: Since the spacecraft is expected to experience extreme temperatures. An active heater is used in the tardigrade payload to keep two small water reservoirs from freezing while in orbit, while thermistors are distributed throughout the spacecraft to measure the temperature [16]. Finite-element analysis and other specialized space thermal studies are conducted to ensure all components can operate as designed.

Structure (STR) Subsystem: The Aluminum 6061-T6 structure for the spacecraft is designed to withstand $12 \mathrm{~g}$ forces in all three axis as experienced under worst-case conditions in a launch vehicle [16]. The structure conforms to the requirements for the P-POD launcher [8] and is used to mount external electronics such as Sun sensor (photodiodes) for attitude determination, solar panels for power generation, and spectroscopy apparatus for the payload.

\section{COMPLEX SYSTEM DESIGN}

The T-Sat project complexity encompasses not only the technical elements, but also the managerial and personnel components.

\subsection{Technical Complexity}

As described in Sec. 2, the technical complexity of the project lies in the interdependencies between all the subsystems. As students apply the design principles learned in the classroom, they get hands-on experience in (i) analysis, (ii) risk assessment, (iii) trade-off studies, (iv) design boundaries, (v) implementation feasibilility, (vi) assembly, integration and testing (AIT), and (vii) documentation.

During the early phases of the design phase, there is a great deal of research into similar projects to learn about the state-of-the-art as well as many lessons from other satellite missions. For many students, the two most challenging components are separating the "needs" from the "wants," and incorporating all the interdependent constraints [17]. This leads to many redesigns as many early ideas are found to conflict with other subsystems. For example, the first designs for the attitude determination and control subsystem included six momentum wheels ( 2 on each axis). However, estimates of the mass, volume, and cost for the wheels forced the team to reconsider and return to a simpler solution consisting of torque rods.

Different designs are evaluated through analysis, risk assessments, and trade-off studies. The analysis accounts for many of the unknowns by incorporating large margins in the early stages of the design and reducing the uncertainty as the designs converge. For example, mass margins of $30 \%$ are used in the preliminary designs and decreased as the project evolves and the subsystems converge on the final designs [18]. The risk assessment considers the "what if" scenarios associated with each option. The risks are mitigated by adding redundancy wherever possible (i.e., torque rods), but most often it is a simplification of designs, making them more robust by removing some of the customizable functionality. Furthermore, each option is also analyzed based on many other parameters such as mass, volume, power consumption, interface options, operating temperature, communication requirements, cost of components, lead time, project schedule, space heritage, documentation available, and even familiarity with products. The large number of parameters and risks associated with each option add to the overall complexity for the project.

As the designs for various subsystems are completed, the students are required to evaluate many of the boundaries between subsystems. For example, in selecting the battery for the spacecraft, the mechanical interfaces must also be considered in order to mount the battery so that it survives vibrations during launch.

The feasibility of each design adds another dimensions that must be considered in the analysis. Students must consider how difficult it is to implement the designs, how to interface other components, and how to access pieces inside a small space within the satellite. The feasibility study leads directly to the assembly, integration, and testing phase of the project, where the complexity of many components, interdependent build schedules, and ability to test designs challenge students to consider many factors simultaneously. Finally, all these studies, analysis, and options considered are documented.

\subsection{Project Management Complexity}

The T-Sat project exposes students to many aspects of project management that deal with the finances and scheduling for the project [19]. 
The monetary exposure requires students to consider the cost of all components, the number of extra components to purchase for prototyping, as well as the cash flow available to decide when to make big purchases without affecting other subsystems. Furthermore, just like the design margins considered in Sec. 3.1, students must evaluate margins for the allocation of funds within the project in order to account for unexpected problems or mistakes. Furthermore, the number of students working on a particular prototype can affect the selection of components purchased. For example, based on the number of software developers on the team, six MSP430 development boards were purchases such that parallel work could be maximized.

The interdependencies in the design play a significant role in the schedule for the project. The Gantt chart for the project includes all the major deadlines from the competition and then works backwards to identify when internal deadlines must be scheduled. Some of the parameters taken into account are exam schedules (as the project is done in addition to the regular class work), lead time for components, availability of manufacturing facilities, and the interdependent integration schedule for the project.

\subsection{Personnel Complexity}

There are more than 100 registered students involved in the project from six faculties (Engineering, Science, Business, Architecture, Art, and Graduate Studies) and 16 departments. The students range from the 1st year through the Doctorate program [3]. Although approximately 40 students are active at any point in time, this still leaves a large group to work on the project. In addition to the students, a team of more than 50 advisors from academia, industry, business, military, government, the amateur radio community, and the community at large play a significant role providing feedback on all elements of the project [20]. The personnel complexity accounts for (i) interactions between stakeholders, (ii) personnel changeover, and (iii) leadership experience.

The daily interactions between stakeholders (studentsstudents and student-advisors) affect the designs considered, risks perceived, and project schedule. The students are organized into teams for each subsystem and payload. The leads for each team compose a systems engineering team that overlooks the entire project. Furthermore, the advisors can be treated as an independent team that provides feedback on high-level options for the project.

Additional challenges come from personnel changeover as students graduate or leave the project for personal reasons and in the development of leadership experience to assess the duration of tasks, division of labor and avoid the rush to implementation without a full planned designed. Furthermore, there are a number of stakeholders such as the University of Manitoba, media, outreach partners, sponsors, and members of the community that also add affect the project through constraints, setting expectations, and defining parameters for the mission. These are representative of high-profile projects in industry where many decisions are scrutinized by the public. Special attention is paid by team leads to maintain the public image for the project and ensure that a unified vision for the entire project is profiled on all fronts.

\section{CONCLUDING REMARKS}

The T-Sat project serves as an extension of the classroom experience that exposes students to many elements of complex system design. The technical complexity of the satellite offers many opportunities for multidisciplinary collaborations to understand the analysis, trade-offs, and risk assessments. The compressed two year schedule challenges students to perform many tasks in parallel while keeping track of manufacturing schedules, lead times for procurements, and availability of cash flow to perform large purchases. The multidisciplinary team with many stakeholders also exposes students to many facets of large industry projects that are otherwise not experienced in the confines of the university classroom. Thus, making the T-Sat project a suitable venue to teach complex engineering systems design, while reinforcing many classroom concepts and a hands-on exposure to systems engineering.

\section{Acknowledgements}

Special thanks to all the T-Sat students and industry advisors, and in particular to the Space Systems team from Magellan Bristol Aerospace.

\section{References}

[1] W. Kinsner, "Challenges in the design of adaptive, intelligent and cognitive systems," in Proc. of the Sixth IEEE Int. Conf. on Cognitive Informatics, pp. 13-25, 2007.

[2] Geocentrix Technologies Ltd., H.R.MacMillan Space Centre, and Jaymie M. Matthews, A proposal to initiate a satellite design challenge for Canadian universities. Vancouver, BC: Geocentrix, January 13, 2009.

[3] Witold Kinsner, M.D. (Ron) Britton, Dario Schor, Arash Fazel-Darbandi, Kris Goodmanson, Cody Friesen, and Emily Bashford, "Preliminary experience from the Canadian Satellite Design Challenge," in Proc. Can. Engineering Education Conf. (St. John's, NL; June 6-8, 2011) 2011.

[4] Dario Schor, Kane Anderson, Arash Fazel-Darbandi, Morgan May, Kris Goodmanson, Brady Russell, Brendan 
Cade, Mohammadreza Fazel Darbandi, Greg Linton, Matthew Woelk, Anthony Schoenfeld, Cody Friesen, Scott McKay, Witold Kinsner, M.D.(Ron) Britton, Malcolm Symonds, Diane Kotelko, Philip Ferguson, Raymond Harris, Zeev Shoichet, Walter Czyrnyj, Samuel Kovnats, and Alan Thoren, "Critical design phase of the T-Sat1 mission," in Proc. 16th CASI Astronautics Conf., ASTRO12, (Quebec City; April 24-26, 2012).

[5] Geocentrix Technologies Ltd., CSDC Rules and Regulations: Mission-level requirement document. Vancouver, BC: Geocentrix, January, 2011.

[6] Geocentrix Technologies Ltd., CSDC Rules and Requirements. Vancouver, BC: Geocentrix, January, 2011.

[7] Dario Schor, Witold Kinsner, and Alan Thoren, "Satellite ground station emulator: An architecture and implementation proposal," in Proc. of the IEEE Canadian Conference on Electrical and Computer Engineering, CCECE 2009, (St. John's, NL; May 3-6, 2009), 2009, pp. 874-879.

[9] Scott McKay, Cody Friesen, Graham Schellenberg, Ben Guest, Alex Paterson, Dario Schor, and Witold Kinsner, "A tardigrade experiment for a nanosatellite," in Proc. of Canadian Space Summit 2011, CSS11, (Calgary, AB: November 23-25, 2011), 2011.

[10] Scott McKay, Cody Friesen, Graham Schellenberg, Ben Guest, Alex Paterson, Dario Schor, and Witold Kinsner, "A prototype for a tardigrade experiment for the T-Sat1 mission," in Proc. 16th CASI Astronautics Conf., ASTRO12, (Quebec City; April 24-26, 2012).

[11] Cody Friesen, Scott McKay, Graham Schellenberg, Ben Guest, Alex Paterson, Dario Schor, and Witold Kinsner, "Solar spectroscopy for the T-Sat1 nanosatellite," in Proc. 16th CASI Astronautics Conf., ASTRO12, (Quebec City; April 24-26, 2012).

[12] Paul White, Trent Erskine, Shawn Koop, Marc Friesen, Kiral Poon, Brady Russell, Mohammadreza Fazel-Darbandi, Joshua Hernandez, David Newsom, Brendan Cade, Dario Schor, and Witold Kinsner, "Design and implementation of magnetic torque rods for the T-Sat1 nanosatellite mission," in Proc. 16th CASI Astronautics Conf., ASTRO12, (Quebec City; April 24-26, 2012).

[13] Brady Russell, Mohammadreza Fazel-Darbandi, Joshua Hernandez, David Newsom, Brendan Cade, Dario Schor, Witold Kinsner, Diane Kotelko, and Philip Ferguson "Implementation of attitude control algorithms for the T-
Sat1 nanosatellite mission," in Proc. 16th CASI Astronautics Conf., ASTRO12, (Quebec City; April 24-26, 2012).

[14] Kane Anderson, Matthew Woelk, Craig Nemeth, Hossain Al-Masroor, Greg Linton1, Frank Serafin, Johnson Vilayvanh, Dario Schor, Witold Kinsner, Diane Kotelko, and Alan Thoren, "Telemetry and Telecommand Architecture for a Nanosatellite," in Proc. 16th CASI Astronautics Conf., ASTRO12, (Quebec City; April 24-26, 2012).

[15] Arash Fazel-Darbandi, Jesse Doerksen, Jason Tayler, Udeesha Annakkage, Kasun Samarasekera, Mohammadreza Fazel Darbandi, Pawel Glowacki, Dario Schor, Witold Kinsner, Diane Kotelko, Philip Ferguson, David Weber, and Samuel Kovnats, "Design and implementation of a power system for the T-Sat1 nanosatellites," in Proc. 16th CASI Astronautics Conf., ASTRO12, (Quebec City; April 24-26, 2012).

[16] Brendan Cade, Morgan May, Anthony Schoenfeld, Raamjee Mailvaganam, Veronica Marriott, David Newsom, Dario Schor, Witold Kinsner, and Paul Labossiere, "Structural and thermal analysis for the T-Sat1 nanosatellite," in Proc. 16th CASI Astronautics Conf., ASTRO12, (Quebec City; April 24-26, 2012).

[17] Wiley Larson, Doug Kirkpatrick, and Jerry Sellers (eds.), Applied Space Systems Engineering, McGraw-Hill, 2009, $895 \mathrm{pp}$.

[18] Rules for the Design, Development, Verification, and Operation of Flight Systems. NASA Goddard Space Flight Center Standard GSFC-STD-1000E, 2009. Available as of March 25, 2012 from http://standards.gsfc.nasa.gov/gsfc-std/gsfc-std-1000/gsfcstd-1000-e-with-administrative-changes.pdf

[19] Julie Chesley, Wiley Larson, and Marilyn McQuade, Applied Project Management for Space Systems, McGrawHill, 2008, 728 pp.

[20] Dario Schor, Kane Anderson, Cody Friesen, Stephanie Reid, Brady Russell, Reza Fazel-Darbandi, Arash FazelDarbandi, Witold Kinsner, M. D. (Ron) Britton, Malcolm Symonds, Diane Kotelko, Philip Ferguson, Raymon Harris, Walter Czyrnyj, Samuel Kovnats, Maia Schor, Alan Thoren, and Brendan Hughes, "Enhanced student performance through industry advisors," in Proc. Can. Engineering Education Conf. (St. John's, NL; June 17-20, 2012) 2012, $4 \mathrm{pp}$. 